\title{
Innovative Design of Intelligent Conference Management Platform
}

\author{
Zehui Liu ${ }^{1}$, Wenli Gong ${ }^{2}$, Jiankang Zhang ${ }^{3 *}$ \\ 1,3 School of International Economy \& Tourism Management, Zhejiang International Studies University, Hangzhou, \\ Zhejiang, 310023, China \\ ${ }^{2}$ School of English Studies, Zhejiang International Studies University, Hangzhou, Zhejiang, 310023,China \\ *Corresponding author. Email: zhangjk@zisu.edu.cn
}

\begin{abstract} of conference participants.

\section{INTRODUCTION}

With the development and progress of science and technology, human beings have entered the era of the mobile Internet to intelligently define future operations, and all industries are facing great challenges. Intelligent services under big data are driven by user needs, and conference system has developed digitally, intelligently, integrated and multifunctionally. The development of intelligent conference management platform will become the future development direction of the conference industry. It integrates online data and offline services into one, which is more helpful to improve conference efficiency.
\end{abstract}

The conference control module, on-site operation module, alliance promotion module and data cloud module are the four major modules of the intelligent conference management platform. The on-site operation module includes common activities and other activities, as well as free and paid functions. Conference promotion method includes micro promotion, media network promotion, SMS + email promotion, etc. The intelligent conference management platform uses "Meeting + Internet" to integrate general control, on-site operations, alliance promotion, and data cloud integration to achieve the combination of online and offline services, which is conducive to improving the operational efficiency of the conference and meeting the various needs

Keywords: Conference promotion, data cloud scenarios, application of iBeacon technology

\section{DESIGN of CONFERENCE GENERAL CONTROL MODULE}

\subsection{Introduction to Conference General Control Module}

The conference general control module will provide platform-based management throughout every link of the entire exhibition event project operation process, and provide a one-stop digital service management SaaS system (Software-as-a-Service) for the conference, including the establishment of a covered network infrastructure and software and hardware operation service platform, which provides overall control of conference affairs data, and controls a series of operation services from pre-meeting to mid-meeting and post-meeting; organizes all mastered resources and data into information systems and provides them to various users use; turning computer resources and applications into various services greatly facilitates users, customized services can be customized by simply operating the service platform through the Internet.

\subsection{Functions of the Conference General Control Module}

The conference general control module holds all the conference related data and provides a variety of functions, including the exquisite responsive theme station function adapted to the PC, iPad, and mobile phone display; it provides a form import / export function that can effectively and conveniently manage the participants; Statistics and analysis of website visits and functions of registration channel sources; functions of distributing vouchers such as participation certificates, real-time tracking and analysis of data such as participants and guests, and data monitoring functions to grasp the status of the event anytime, anywhere; provide paid tickets, free tickets, Member tickets, VIP tickets, and other multi-ticket functions that support custom ticket types [1];real-time, stable, efficient, intelligent, caring and complete Tips notification service, effectively improving the Tips management function of the participating experience; WeChat mini-programs, PC online, Offline sign-in and other forms of QR code e-vouchers supported by one-code scan-code sign-in function; unified settlement of fees generated by user registration, participation in activities, and sales to the public account autonomous collection function of the designated public account; The 
dimensional code electronic voucher implements the scenario management function for the management and statistics of the scenario service throughout the entire activity.

\section{ON-SITE OPERATION MODULE DESIGN}

\subsection{Field Operation Module Introduction}

The on-site operation module can connect the WeChat wall of each participant to achieve cross-screen interaction. Through this module, it can integrate the conference activities with the WeChat public platform, and easily build the user's own WeChat operation platform. The way articipants follow the public platform to join the game can promote the public platform to be promoted and increase its popularity quickly, and it can turn offline users into online and offline coexisting users, which helps to connect with old customers and develop new customers; participants use WeChat Accounts to participate in live events through WeChat public platforms, computers, and large screens; large screens display live interactive information in real time, so that everyone has a stronger sense of participation; cool large screens, real-time scrolling of information, and a sense of technology, It can stimulate the active participation of field personnel and increase the value of field interaction.

\subsection{Field Operation Module Activity Form}

The common activity forms of the on-site operation module include shake to PK, shake to give away a red envelope, live broadcast of the meeting point, through WeChat to be on the wall, interactive lottery, group purchase crowdfunding, auction second auction and other activity forms that can be easily used on the WeChat public platform. Guests can be invited through the WeChat public service number to accept participants' registration before the meeting, and provide WeChat collection services for ticket sales, and then generate a QR code for sign-in; conduct lotteries, games and other interactions during the meeting, focusing on the WeChat service number, then, you can use WeChat shake, iBeacon, live broadcast, smart wifi and other functions to flexibly dock at the meeting site. In addition, the on-site operation module can innovatively use WeChat conference sales modes such as spike, order, on-site group purchase, and on-site auction to increase sales for users. At the same time, they will expand their publicity efforts and gain more fans, that is, online users. Participants can participate through WeChat and experience convenient and fast WeChat payment collection, which can effectively improve the participation and experience of participants.

\subsection{Field Operation Module Function}

\subsubsection{Free functions}

Free functions include conference creation, registration, conference (ticketing) promotion, conference sign-in, live interaction, data management, data analysis, and online recharge.

Meeting creation is the most basic and main resource in free features. You can use Evolution to create meetings, invite others to meetings, and book the necessary resources for meetings. The specific operations are as follows: (1) Select "File"-> "New"-> "Meeting". A "Schedule" window is displayed, which is similar to the window displayed when you select "File"-> "New"-> "Schedule", but with two additional tabs: "Schedule" and "Meeting ".(2) Enter the appropriate details in the Appointments, Memos, and Recurring tabs of the Appointment window as described in Creating Individual Appointments and Creating Recurring Appointments.(3) To enter detailed information about the meeting, click the Meeting tab to display the Meeting tabbed section.(4) Select the meeting organizer from the "Organizer" drop-down list, and Evolution will automatically enter the current user name in the "Organizer" text box; if you have multiple Evolution accounts, you can select the appropriate account from the drop-down list .(5) Press Enter to add a new entry to the participant list. To delete a participant from the list, rightclick the entry in the participant list and select "Delete" from the pop-up menu; click "Invite Others "button to create a new entry in the table; Evolution also displays the" Select Contacts from Address Book "dialog box where you can select attendees.(6) Click the "Schedule" tab to display the "Schedule" tabbed section. You can use the "Schedule" tabbed section to indicate when all participants are free to attend the meeting and the necessary resources are available The period of the meeting. After the conference is created, registration, registration (ticketing) promotion, and conference sign-in will become very convenient [2].

\subsubsection{Paid functions}

Payment functions include QR code scanners, label printers, and iBeacon devices, which are increasingly used by conference intelligent hardware, autonomous account collection (WeChat + ticket collection), conference platform ticket collection, and public account independence Card vouchers (one-click deposit of etickets into WeChat card package),shaking of WeChat to send red envelopes (public account increase powder) and functions supported by field personnel.

Leasing and sales of conference function hardware is one of the main profit models of intelligent conference management platforms. The intelligent conference exhibition board can push multimedia content, including video, audio, pictures, subtitles, Office documents, PDF 
documents, etc., to a variety of designated display terminals, such as high-definition LCD screens and touch interactive screens, via the network, according to the specified display template Play and realize the whole process management of multimedia content distribution. Intelligent conference display boards have the following advantages: (1) a complete digital display board management solution. Provides a series of complete digital exhibition board management and playback solutions such as media content, playback equipment, playback templates, playback plans, and playback monitoring.(2) Centralized management and flexible operation [3].Centralized control of media content, playback equipment, templates and plans, network push, devices can be managed in groups, and system interface operations are flexible and convenient.(3) Various optional playback devices. Supports Linux, Windows kernel, and Android and iOS platforms. You can use a separate player to connect high-definition TVs and screens. You can also use smart TVs or iPAD tablets. (4) Suitable for different business scenarios. Support local and remote playback, remote playback is real-time and efficient. The local playback is transmitted once and played repeatedly, which is stable and reliable. It is suitable for the application scenarios where the content is repeated and the stability is given priority.

\section{ALLIANCE PROMOTION MODULE DESIGN}

\subsection{Introduction to Alliance Promotion Module}

The alliance promotion module follows the development direction of the future advertising media industry. It is an innovative digital accurate promotion model. The organizer creates tasks, creates links, sets rewards, uses the push platform to generate links, calculates revenue, establish a cooperative relationship with partners directly or indirectly, and then output links, obtain benefits, and further promote links to discover more potential customers, obtain better cooperation with them, and establish good reciprocal relationships. The alliance promotion module has the following advantages: Under the influence of the social recommendation system of the Uber model, the fission propagation ability of the community system has been continuously improved; $100,000+$ partners and a large V resource bank have provided a powerful reward and settlement system; the MICE Social Marketing Alliance covers real estate, automobiles, catering, fast food Sales and other industries are more accurate and effective than traditional methods such as email, phone, and banner advertising; and the integration of marketing and sales promotion and the integration of online and offline shows a trend.

\subsection{Ways of Alliance Promotion}

\subsubsection{Micro promotion}

With its own professional and systematic promotion mechanism, the organizer can use the promotion cloud platform to get the attention of WeChat resources (individual / public account) in many industries, and its generous reward mechanism can stimulate the promotion effect, reach more audiences, and greatly improve Admission check-in rate, efficient and worry-free [4].

\subsubsection{Media network promotion}

With the help of media resources with cooperative relationships, analyze the current consumer consumer psychology and be guided by the current user needs, conduct targeted and age-specific publicity and promotion, enhance the flexibility and reliability of media network promotion, and improve the media network promotion. Effective coverage and timely adjustments to media network promotion strategies.

\subsubsection{SMS +email promotion}

Using text message and email promotion methods, the organizer and the participants are connected in the most basic way, and more effective, professional and efficient communication of effective information is provided to assist the organizer to get in touch with the participants and obtain certain benefits.

\section{DATA CLOUD MODULE DESIGN}

\subsection{Introduction to Data Cloud Module}

The data cloud module will provide customers with onestop data monitoring statistics and agile data analysis services, that is, the entire process of data monitoring and analysis to guide operation management. Provides scientific management and analysis of data for customer's activity management, providing customers with Activity optimization provides more effective, reasonable, and scientific advice [5].

\subsection{Data Cloud Module Features}

First of all, it has the characteristics of data and can provide one-stop activity-based data management and monitoring services; it also has the characteristics of intelligence, can understand the connection between data, perform intelligent scientific analysis, find important opportunities and optimize and improve services 
experience; it also has customized features, that is, it can provide exclusive customized data intelligent analysis operation management service solutions [6].

\section{SUCCESSFUL CASES}

Huidian.com, is a well-known digital conference service platform in China. Meeting Point Network uses WeChat shake, live broadcast, conference promotion, cloud data and other technologies to provide customers with preconference, during- conference and post-conference digital invitations, live voting, sweepstakes, push conference reports, comprehensive data analysis, etc.

\subsection{Sogou Mobile Marketing National Summit}

With the technical support of the conference network, Beijing, Shanghai, Guangzhou, and Chengdu jointly launched the Sogou Mobile Marketing National Summit. Huidian.com cooperates with Sogou Search to provide a digital conference management platform for the Sogou National Marketing Summit, assist Sogou to notify participants before the meeting, generate a QR code as an electronic voucher, and sign in with a one-digit scan code. Participants conducted interactive lottery, provided technical staff support for the entire conference process, conducted statistical analysis of the data after the meeting, and fed it back to Sogou. This effectively promoted the process of the Sogou Mobile Marketing National Summit, provided it with intelligent integrated operation services, and helped It forms big data assets.

\subsection{Beautiful China Shenzhen Charity Dinner}

The Beautiful China Shenzhen Charity Dinner was held as an important content of the 5th "China Charity Charity Project Exchange Exhibition". The conference network provided intelligent and integrated digital conference management for the dinner: SMS reminders to participants before the conference, distribution the two-dimensional code serves as the electronic voucher for the conference. At the meeting, the two-dimensional code is checked in and verified at the scene, a series of labels are printed, the media is promoted on the dinner site, and the technical staff is provided with guidance on the site. From the invitation to the end of the event, the intimate one-stop service experience allows the conference science and technology innovation services and humanistic care to accompany you along the way.

\section{CONCLUSION}

Using the way of "Meeting + Internet", the combination of online and offline services will integrate general control, field operations, alliance promotion, and data cloud integration into one, providing professional tools and supporting human services to help organizers form big data assets; not waste every customer, dig more user value, provide users with personalized services, let users feel the humanity of the service, and let the service reflect the user's personalization. Promote the conference process, improve the efficiency of the conference operation. It improves the participants' a sense of experience, creating an integrated conference operation service platform, and one-stop intelligence to meet the needs of the organizers.

\section{REFERENCES}

[1] Yan, S. H. Problems and Countermeasures of Exhibition Industry Development Under the Support of Network Technology J. Economic Development Research, 2016(02), p. 220.

[2] Gong Tao. Digital platform enhances smart services [j]. China Property Management,2013 (09), pp.20-23.

[3] Hao, R. Research on the Development of Exhibition Economy Under the Internet Technology J. China Business \& Trade,2015(01), pp.140-141.

[4] Shi Hongwei. Problems and countermeasures in the information management of conventions and exhibitions. Western Leather, 2016(04), p.110.

[5] Zhu Jian, Lu Xinxin.How to Reform the Practical Teaching of "Conference Operation Management" Course. Cultural Education Materials,2018(9), pp.181182.

[6] Hao Haiyuan. Analysis of the current status of the conference o2o platform in China. Modern Business, 2015 (18), pp.43-44. 